\title{
ENCAPSULATED PAPILLARY CARCINOMA CONCOMITANT WITH AXILLARY METASTASIS OF NEUROENDOCRINE CARCINOMA - CASE REPORT
}

Juliana Lopes de Aguiar Araujo', Ubiratan Wagner de Sousa1', Lourdes Maria Dantas de Góis², Ana Tereza Diniz Marinho de França²

1 Liga Norte Rio-Grandense Contra O Câncer - Natal (RN), Brazil.

2Universidade Potiguar - Natal (RN), Brazil.

Introduction: Encapsulated papillary carcinoma corresponds to approximately $1 \%$ of breast tumors. Although rare, it has a good prognosis, slow growth, and a 10-year survival close to $100 \%$. Axillary metastases of non-breast neoplasms are also uncommon, and the coexistence of metastatic non-breast neoplasm in the axilla and another histological type of breast neoplasm is exceedingly rare. Objective: To describe a case of breast neoplasm with axillary metastasis of non-breast disease. Method/Case report: F.R., 95 years old, had a nodule in the left breast (LB) for 1 year, with progressive growth. Examination revealed an irregular nodule in the entire LB with retraction of the papilla, measuring $11 \times 12 \mathrm{~cm}$, and hardened lymphadenopathy of $2 \mathrm{~cm}$ in the right axilla. Ultrasound showed a mixed tumor with a multinodular central mass of $18.3 \times 19.7 \mathrm{~cm}$ and two enlarged, heterogeneous lymph nodes in the right axilla. Core breast biopsy indicated carcinoma with a papillary growth pattern. She underwent left mastectomy and lymph node excision in the right axilla. Anatomopathological results revealed a $14 \mathrm{~cm}$ encapsulated papillary carcinoma with free margins and right axillary lymph node as a high-grade metastatic neuroendocrine carcinoma. She is on exclusive hormone therapy with tamoxifen. When investigating the primary axillary site, abdominal computed tomography (CT) showed an expansive, predominantly cystic, retroperitoneal formation on the left side, in close contact with the tail of the pancreas, adrenal gland, and left renal vein, pending clarification, probably related to the right axillary lesion. Due to clinical conditions, she continues without a diagnosis for the primary site of axillary metastasis. Results/Discussion: Encapsulated papillary carcinoma is characterized by being solitary, unilateral, of slow growth, with papillary proliferation, central malignancy, surrounded by cystic ductal dilatation. It has a good prognosis and is rarely metastatic. Usually, it presents low or intermediate nuclear grade and strong positivity for estrogen receptor. Neuroendocrine tumors are a relatively rare group of neoplasms in any part of the body, occurring in the digestive tract in $62 \%-82 \%$ of cases. They can be asymptomatic or have symptoms associated with the primary tumor or metastasis. They are a heterogeneous group, often found in the retroperitoneum, abdominal lymph nodes, and mediastinum, usually have multiple metastases, and their primary site is hard to identify. Axillary metastases are uncommon, mainly coexisting with breast neoplasm of good prognosis. Conclusion: The rarity of axillary metastasis of neuroendocrine carcinoma associated with the good prognosis of encapsulated papillary carcinomas gives scientific importance to the current case since its axillary approach allowed reaching an unlikely diagnosis of neoplasm with uncommon metastatic site. 